\title{
Validity and reliability of a locomotor stage-based functional rating scale in spinal cord injury
}

\author{
B Maurer-Burkhard ${ }^{1}$, I Smoor ${ }^{1}$, A von Reumont ${ }^{1}$, G Deckstein ${ }^{2}$, I Stierle ${ }^{1}$, R Rupp ${ }^{1}$ and C Schuld ${ }^{1}$
}

Study design: This is a prospective observational cohort study.

Objectives: The objectives of this study were to apply and adapt a rating scale based on locomotor stages (LSs) derived from cerebral palsy (CP) to spinal cord injury (SCl) and to quantify its inter-rater reliability and construct validity.

Methods: The inter-rater reliability of LSs originally developed for children with $\mathrm{CP}$ was tested in a chronic SCI cohort. On the basis of the distribution of the LSs for CP, Locomotor Stages in Spinal Cord Injury (LOSSCI) were defined. Their validity was then tested with the Spinal Cord Independence Measure (SCIM) in another acute SCl cohort.

Results: The 10-point LSs for CP were assessed by two raters in 65 chronic patients. Weighted Cohen's kappa (WCk) was 0.985 $(P<0.0001)$. Only four mismatches were found, resulting in an accuracy of $93.4 \%$. On the basis of the distribution of the LSs for CP in $\mathrm{SCl}$, the five-point LOSSCI grading scale was developed. WCK of LOSSCI was $0.976(P<0.0001)$. Only three mismatches between raters were found, resulting in an overall accuracy of $95.1 \%$. The validity data sets consisted of 448 SCIM records from 161 patients obtained within the first year after injury. Spearman's correlation coefficients were the highest between LOSSCI and SCIM indoor mobility (room and toilet; $R=0.82$ ) and the lowest between LOSSCI and SCIM respiration and sphincter management $(R=0.68)$.

Conclusion: LOSSCI provides a reliable and valid clinical tool to assess locomotor function in SCI. LOSSCI not only reflects bipedal walking but also covers a wide range of key motor skills.

Spinal Cord (2016) 54, 619-625; doi:10.1038/sc.2015.223; published online 12 January 2016

\section{INTRODUCTION}

During the past decade, novel assessments focusing on different aspects of spinal cord injury (SCI) were developed to obtain good psychometric properties for clinical trials. These multidimensional functional scores such as Spinal Cord Independence Measure (SCIM) ${ }^{1}$ or Graded Redefined Assessment of Strength Sensibility and Prehension $^{2}$ have been developed as outcome measures specifically for the SCI population to be used to quantify the effects of novel therapeutic interventions. ${ }^{2}$ Although fulfilling the needs of researchers in terms of having sound psychometric properties, they have gained less acceptance among clinical practitioners because of their complexity, time required to perform the assessments and nonintuitive interpretation. Examples of clinically well-accepted scores are the Frankel scale ${ }^{3}$ and its successor the American Spinal Injury Association (ASIA) Impairment scale (AIS), ${ }^{4}$ which both classify patients on a five-point scale in an ascending order according to the extent of the neurological impairment caused by the SCI. Their regular clinical use is mainly based on their good clinical interpretability and easiness of being communicated to others. Each grade represents a distinct clinical impression of a patient; for example, patients with a complete lesion are unable to walk and have a poor prognosis to recover substantial motor and sensory function. Although distinct categories exist for the classification of the neurological impairment, their counterparts for categorization of the overall functional status of a patient are missing. The need for a functional classification scheme based on a limited number of grades was addressed recently by the introduction of the SCIM grades. ${ }^{5,6}$ The SCIM grades consist of eight clusters describing distinct patterns in SCIM's 17 subitems, which were derived by a purely data-driven approach ( $k$-medoides cluster analysis). The respective grades represent rather abstract values without a direct clinical relevance and are difficult to communicate to professionals and patients. The Walking Index for Spinal Cord Injury Version II (WISCI $)^{7}$ is another frequently used capacity measure of walking function for use in clinical trials, which captures the extent and nature of assistance that persons with SCI require to walk. ${ }^{8}$ By design, it captures only walking function and not overall functional status, and it accordingly has floor effects for nonambulating subjects, as there is only one level available for grading nonambulating subjects.

A different approach to setting up a grading scheme for describing pathological motor function is based on specific locomotor stages (LSs) developed for infants suffering from cerebral palsy (CP) by Vojta. ${ }^{9}$ Respective stages capture in an ascending order: initiation of a targeted movement, locomotion requiring the use of the arms and legs, bipedal ambulation, and a one-legged stand for $>3 \mathrm{~s}$ (Stork test). ${ }^{10}$ They represent a hierarchical 10-point scale, with every stage having a clear, clinically meaningful description of specific locomotor patterns. ${ }^{11}$ The progress of an individual with SCI during rehabilitation is to some extent comparable to the typical development of ontogenic locomotion in children. Normative studies in the first half of the twentieth century provided detailed, qualitative descriptions of ordered sequences of stages for prone and upright locomotion..$^{12,13}$ The recovery of ambulation after $\mathrm{SCI}^{14}$ also represents a hierarchical

${ }^{1}$ Heidelberg University Hospital, Spinal Cord Injury Center, Heidelberg, Germany and ${ }^{2}$ Department of Orthopaedics, Werner Wicker Clinic, Werner Wicker KG, Hessen, Germany Correspondence: C Schuld, Heidelberg University Hospital, Spinal Cord Injury Center, Schlierbacher Landstraße 200a, Heidelberg 69118, Germany.

E-mail: Christian.Schuld@med.uni-heidelberg.de

Received 18 March 2015; revised 15 October 2015; accepted 21 October 2015; published online 12 January 2016 
process and follows to some degree distinct stages ${ }^{15}$ - for example, a certain degree of trunk stability is a prerequisite for the ability to stand, which is a precondition for achieving independent ambulation.

Therefore, the aim of this project was to apply and adapt LSs derived from CP to SCI and to quantify the inter-rater reliability and validity of this rating scale. We hypothesized that despite the different underlying pathophysiological mechanisms of CP and SCI the LSs originally designed for classification of CP can be adapted to SCI with sound (validity correlation coefficient $>0.7$ (Fitzpatrick et al. ${ }^{16}$ ) and reliability correlation coefficient $>0.7$ (Aaronson et al. ${ }^{17}$ )) psychometric properties.

\section{MATERIALS AND METHODS}

As a first step, the 10 LSs (Table 1) originally developed for the assessment of children with CP were assessed in an SCI cohort. The aim of this initial pilot study was twofold: (1) to examine the basic applicability and the distribution of the LSs for CP in a typical SCI cohort and to (2) test the inter-rater reliability in individuals with SCI. On the basis of the results of this pilot study, an expert-based consensus process was initiated to adapt the CP LSs for consistent classification of the motor skills of individuals with SCI.

At the same time, a validity trial was conducted in a separate SCI cohort at a different SCI center. In the framework of this trial, study participants were assessed with the original LSs for CP. However, the validity analysis was performed with the SCI-adapted LSs.

\section{Pilot study on applicability and reliability in SCI}

The inter-rater reliability of the original LSs was conducted in 2006 at the Spinal Cord Injury Center, Werner Wicker Clinic, Bad Wildungen, Germany by two trained ( 1 day of training in theoretical and practical issues, including supervision) physical therapists. Sample size was determined before study onset on the basis of reliability study correlation coefficients of the International Standards for Neurological Classification of Spinal Cord Injury (ISNCSCI), ${ }^{4}$ the Functional Independence Measure ${ }^{18}$ and the Barthel Index. The planned sample size of approximately 70 patients was calculated on the basis of an estimated correlation between raters of at least $0.8 \pm 0.1$ of the above-mentioned established scores. Inclusion criteria were subjects at least 8 weeks after SCI (without any maximal time since injury constraint), aged between 18 and 80 years and two successive assessments by two raters within 1-5 days. The study protocol was approved by the local ethics committee (application number: 15/06). All participants gave written informed consent.

\section{Validity trial}

Two databases were combined for the evaluation of the construct validity. The local Heidelberg data sets of the European Multicenter Study on Human Spinal Cord Injury (EMSCI) $)^{14}$ provided the data source for the SCIM. ${ }^{1}$ EMSCI uses a fixed time schedule referenced to the date of injury. It is tested within $2,4 \pm 1.7$, $12 \pm 2,24 \pm 2.6$ and $48 \pm 7.1$ weeks after injury (WAI). The SCIM assessment was chosen as a gold standard and reference for the validity determination, because it is thoroughly investigated and it documents functional scores covering many functional aspects of daily living in SCI subjects.

\section{Table 1 Locomotor Stages in Spinal Cord Injury (LOSSCI) transformation table}

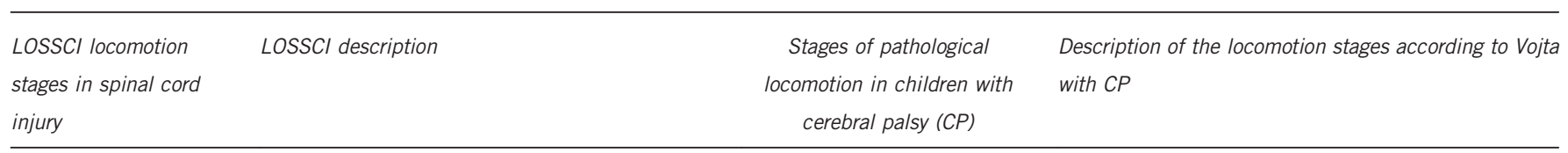

Orienting to and touching/grasping an object may be possible in supine position, no trunk uprighting in prone position

Trunk uprighting in prone position through propping on arms; supporting on one arm and reaching/ grasping with the other is possible

Creeping: locomotion in prone position using the arms

Quadrupedal locomotion using the arms and legs, either crawling or in the vertical position (cruising around furniture)

Pathological mass movements, gaze fixation and following or touching/grasping an object is absent.

2

3

4

5

6
Pathological orientation to an object, gaze fixation and following with head and eye movement or touching/ grasping an object with a hand (in supine).

Pathological trunk uprighting in prone position, propping on both arms to orientate or propping on one arm and reaching/grasping with the other.

Pathological creeping: locomotion in prone position using the arms, with or without using the legs.

'Bunny-hopping': pathological homologous locomotion in 4-point kneeling.

Pathological cross patterned crawling with either three or two supporting extremities.

Verticalized quadrupedal locomotion in cross-pattern using the arms for support-cruising, walking with walking aids.

Independent pathological walking on flat surfaces, stopping on command-maximum one extra step. Walking on slopes without walking aid is possible.

One-legged stand-right or left longer than $3 \mathrm{~s}$.

One-legged stand-right and left longer than $3 \mathrm{~s}$.

LOSSCI stages and descriptions are depicted on the two most left columns. The original locomotion stages are shown in the two most right columns.

Mass movements: coordinated movements still in a developmentally low differentiation level; Creeping: locomotion in prone position using the arms with/without the use of legs, stomach on floor, for example, commando crawling; Crawling: locomotion on hands and knees. 
LSs were assessed by 10 trained physical therapists from 2002 to 2011 in Heidelberg. LSs were documented for each of the study participants every 4 weeks. Data sets from both sources were matched based on the rule that respective assessment dates were not allowed to differ by $>15$ days. This threshold was chosen as a compromise between a high sample size and a tolerable probability of functional or neurological changes that can occur in the acute and subacute rehabilitation phase time window. The EMSCI protocol was approved (S-188/2003) by the ethics commission of the medical faculty of the Heidelberg University.

\section{Spinal Cord Independence Measure}

The SCIM is a comprehensive ability rating scale specifically for SCI individuals. It captures independence on performing everyday tasks in 17 items categorized into self-care, respiration and sphincter management, mobility (indoors and outdoors, on even surface) and mobility outdoors.

The SCIM was revised in 2001 (SCIM II ${ }^{1}$ ) and in 2007 (SCIM III $^{5}$ ). In SCIM III, single items were changed or deleted, but the category and total sum scores remained unchanged. Accordingly, EMSCI switched from SCIM II to SCIM III in 2007. The majority of subjects in the validity cohort were assessed with the SCIM II (76.4\%); 23.6\% were scored with the SCIM III.

\section{Statistical analysis}

Accuracy, defined as the percentage of raw agreement between raters and Cohen's weighted $\kappa$ coefficients, was used to quantify inter-rater reliability. Validity was evaluated by Spearman's rank correlation coefficients, which are interpreted as weak (0.2-0.4), moderate $(0.4-0.7)$, strong $(0.7-0.9)$ and excellent $(0.9-1.0) .{ }^{19}$ The threshold for significance was $\alpha<0.05$. Statistical analysis was performed with $\mathrm{R} 3.1 .2 .^{20}$

\section{RESULTS}

\section{Applicability}

The original LSs were assessed in 65 patients (77\% male, $43.1 \%$ tetraplegia, $49 \%$ motor and sensory incomplete lesion). Mean age was $44.9 \pm 16.0$ years, and the time between injury and testing was $10.8 \pm 7.6$ years. The two raters conducted most $(35.4 \%)$ of the assessments per individual within 1 day, with a median of 2 days.

The LSs for CP have a nonuniform distribution in the assessed SCI population (Figure 1a). Grades 0 and 4 are missing and the upper stages $\geqslant 6$ are sparsely represented.

\section{Reliability of the original LSs}

Weighted Cohen's kappa was $0.985(P<0.0001$, confidence interval (CI): 0.968 1.000). Only four mismatches between raters were found, resulting in an accuracy of $93.4 \%$ (CI: $84.1 \%, 98.2 \%)$.

\section{CP LS adaptation to SCI}

On the basis of expert consensus, the 10-point scale of the LSs as described by Vojta for $\mathrm{CP}^{11,21}$ were adjusted for the SCI population. The consensus process was twofold. First, an expert panel consisting of all authors provided the conceptual basis for the adaptation of the LSs. Among the seven experts were five experienced physical therapists (BM-B $>25$ years in SCI medicine, Imke Smoor $>5$ years, AvR $>20$ years; MSc in physical therapy, Irma Stierle $>30$ years, GD $>10$ years; MSc in physical therapy) and two experienced researchers (RR $>15$ years; PhD in Biomedical Engineering, CS $>10$ years; MSc in Medical Informatics). The main outcome of the expert panel discussions was the need to adapt the CP LSs to properly reflect functional deficits related to a spinal cord lesion. As a result, stages mainly determined by impairments of higher cerebral function-particularly relevant in children with $\mathrm{CP}$-were omitted or pooled, with adjacent stages primarily focusing on locomotor function. In a second step, the distribution of the original LSs for CP (refer to 'Applicability' section) of the applicability cohort was analyzed for the final transformation.
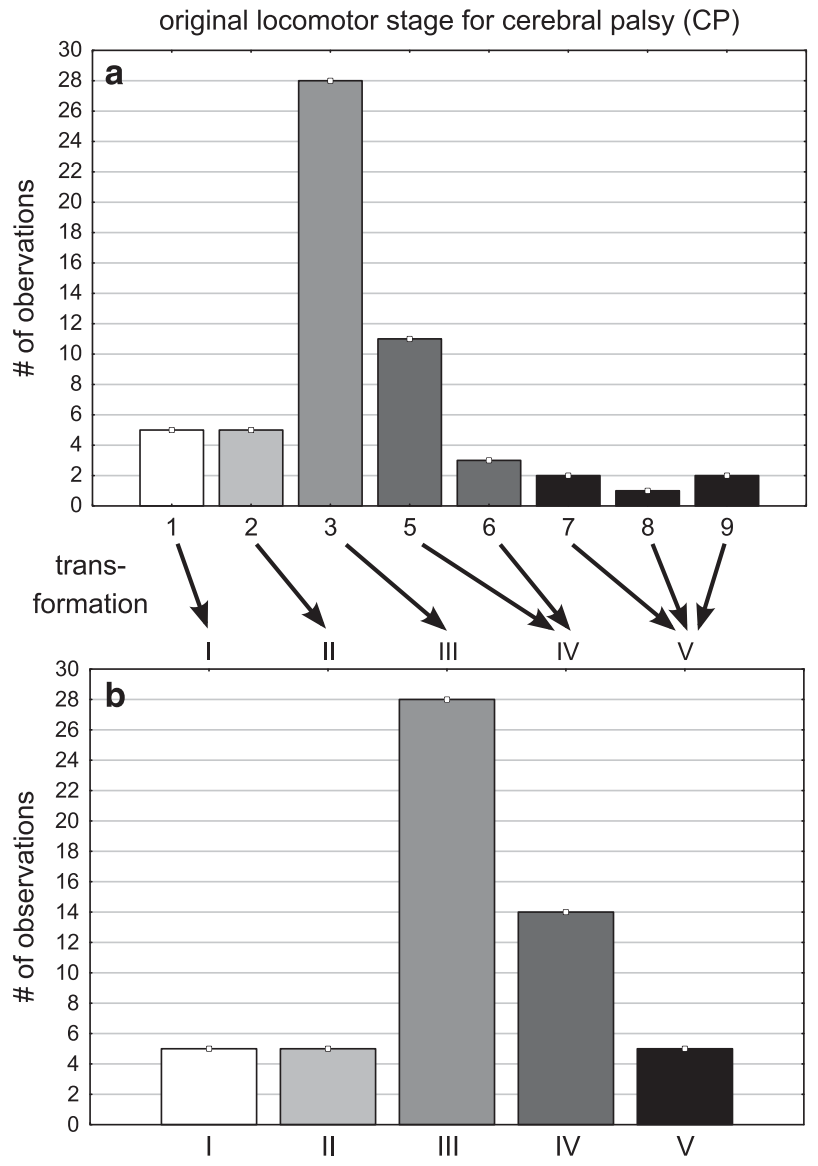

transformed locomotor stages for spinal cord injury $(\mathrm{SCl})$

Figure 1 Applicability study results. (a) Distribution of the original LSs developed for CP. (b) Distribution after transformation of the data set (a) into the transformed LSs for SCl. The transformation is (1) color-coded and (2) indicated by arrows between panels (a) and (b). Stage 4 of the original LSs was not found in the $\mathrm{SCl}$ cohort, and it was accordingly omitted in panel (a).

The composition and description of the Locomotor Stages in Spinal Cord Injury (LOSSCI) stages were iteratively modified to achieve a distribution as uniform as possible with the constraint of avoiding too many subjects classified with the lowest and highest LOSSCI stages (ceiling and floor effects). A statistician from the Medical Biometry Department of Heidelberg University Hospital supported this step. Individuals with SCI were not consulted in the decision-making process because of their lack of expert knowledge in the field of locomotion assessment.

The adapted five-point scale was named LOSSCI (Figure $1 \mathrm{~b}$ and Table 1) and subsequently analyzed in terms of reliability and validity. The original stage $0\left(\right.$ Vojta $\left.^{11}\right)$ tests the ability to initiate face-to-face contact. As this ability is generally not impaired in individuals with SCI, stage 0 was omitted and stage 1 was transferred to LOSSCI I.

Stage 4 was omitted, because this LS for CP does not exist in the SCI cohort. Patients of stages 5 and 6 need the help of upper-limb function to achieve locomotion and were therefore fused into one group (LOSSCI IV). With respect to activities of daily living and independent locomotion, the ability to perform a one-legged stand was not considered relevant enough to define a separate LS. Therefore, stages 7-9, which are defined by the ability to walk independently and to perform a one-legged stand, were combined with LOSSCI V. 


\section{Functional description of the LOSSCI stages}

LOSSCI I tests the ability to control the position of the body so that it is possible to carry out isolated goal-directed movements of the arms. LOSSCI II tests the ability to upright the trunk against gravity in prone position and to perform isolated goal-directed movement with one arm. LOSSCI III tests locomotion in prone position using either the arms or the arms and legs (creeping-stomach remains in contact with the ground). LOSSCI IV tests the ability to move the body forward either by crawling (support on the hands and knees-stomach not on the ground) or by a verticalized bipedal gait with support through the arms using a walking aid, such as crutches or a wheeled walker. Substantial arm function is mandatory in LOSSCI $\mathrm{IV}$, and its presence is the main discriminating factor between this stage and the highest stage. LOSSCI V represents the ability to walk bipedally without walking aids and the ability to perform a one-legged stand (this is considered a higher skill important for walking up and down the stairs independently).

\section{Assessment procedure}

Each LOSSCI stage should be evaluated in ascending order. The LOSSCI grading finally assigned to an examined subject is determined by the highest stage this subject can accomplish. This means that for testing LOSSCI I-III the subject is placed in the prone position on the treatment table. To qualify for LOSSCI III or IV, the subject has to creep/crawl for one full cycle using both arms, thereby fulfilling the criteria for quadrupedal locomotion. Physical assistance is not allowed during assessment. The need for walking aids such as braces (except ankle-foot orthosis), crutches or walkers for bipedal locomotion discriminates between LOSSCI IV and V. Someone who needs walking aids to achieve assisted bipedal ambulation is graded as LOSSCI IV. If no walking aids beside an ankle foot orthosis are needed for independent bipedal locomotion, a subject is graded as LOSSCI stage V. Experienced therapists may start the assessment testing the most probable stage. However, it is still recommended to test and document the examination results of all stages and not just test for the highest possible stage, which in the end determines the LOSSCI stage. With this approach, a subject with central cord syndrome and the ability to walk has to be graded as LOSSCI IV. For better comprehensibility, it is recommended to document that the LOSSCI I and II criteria are fulfilled but not the LOSSCI III criterion.

Example 1: Neurological Level of Injury (NLI) C5, AIS A.

A 31-year-old man with a complete cervical SCI caused by a car accident was assessed at all five EMSCI time frames during the first year after injury using the ISNCSCI, SCIM and LOSSCI assessments. The NLI fluctuated between C4 and C5 (C5, C5, C4, C5, C4). The AIS grade was $\mathrm{A}$ at all assessments. The upper-extremity motor scores (UEMS) were 14, 14, 18, 20 and 20. The lower-extremity motor score (LEMS) remained at 0 . The total SCIM score was successively 0,13 , 17, 18 and 12 (deterioration was because of loss in bladder and self-care function). LOSSCI was graded as I at all assessments, because the subject was not able to upright the trunk in prone position through propping on arms (LOSSCI II) or to move forward using just his limited arm function (LOSSCI III).

Example 2: Central Cord Syndrome incomplete C4 lesion.

The course of recovery of a 64-year-old man with an incomplete cervical lesion for the five EMSCI time frames was as follows: NLI: C4, C4, C5, C5, C4; AIS: C, D, D, D, D; UEMS: 12, 20, 37, 37, 41; LEMS: 10, 30, 47, 47, 47; SCIM: 10, 19, missing, 69, 81. Besides the first assessment, the LEMS is approximately 10 points higher than the UEMS, indicating a central cord syndrome lesion. ${ }^{22}$ Three LOSSCI assessments are available for the first (LOSSCI I), the second (LOSSCI II) and the fourth (LOSSCI IV) time frame. LOSSCI V was not reached, because a one-legged stand was not possible for $>3 \mathrm{~s}$.

\section{Reliability of LOSSCI}

Weighted Cohen's kappa was $0.976(P<0.0001$, CI: 0.948, 1.000). Only three mismatches between raters were found, resulting in an overall accuracy of $95.1 \%$ (CI: 86.3\%, 99.0\%). Two mismatches were found for LOSSCI stage II, which were classified one time as LOSSCI I and as LOSSCI III, respectively, by the second rater. The third discrepancy was found for LOSSCI IV, which was rated as LOSSCI III by the other rater.

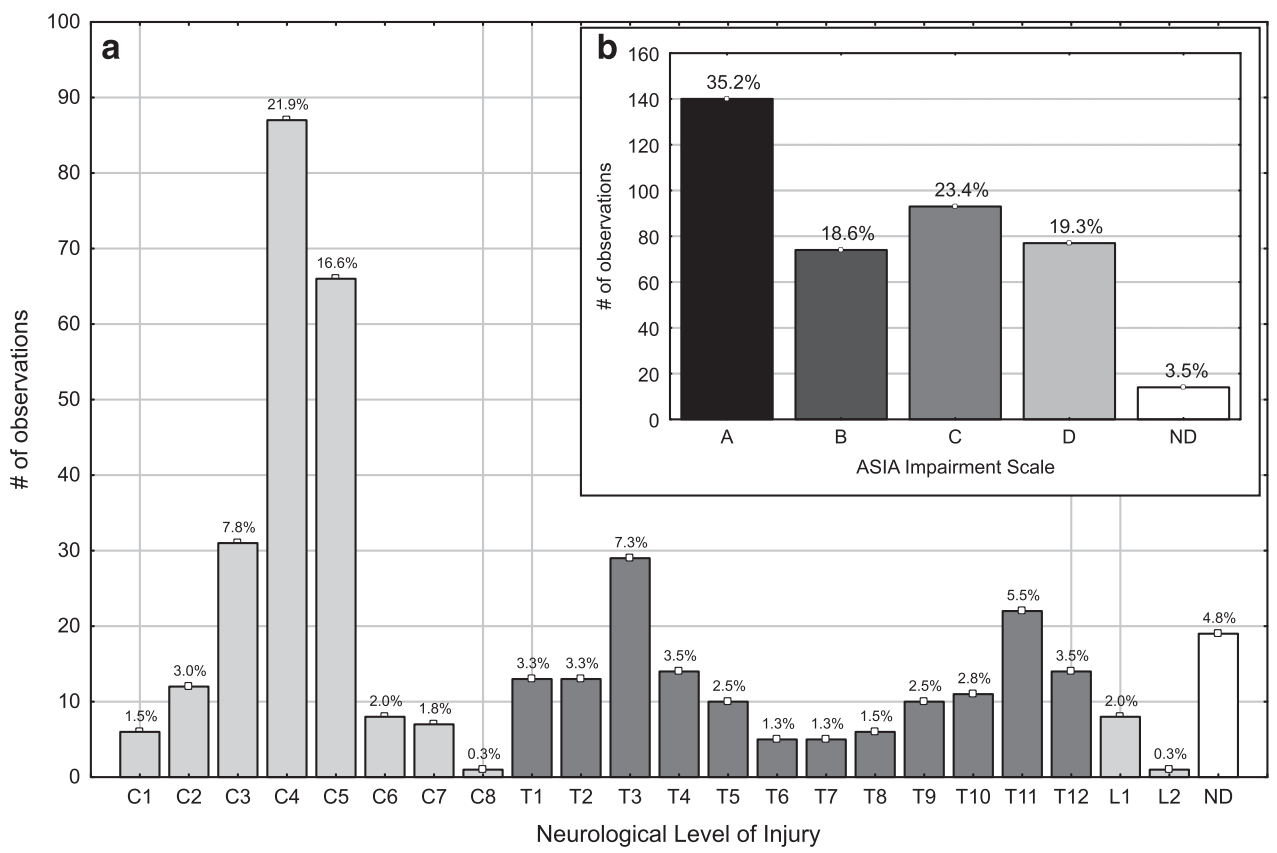

Figure 2 (a) Distributions of the NLI and (b) AIS in the validity cohort. ND, not determinable. 


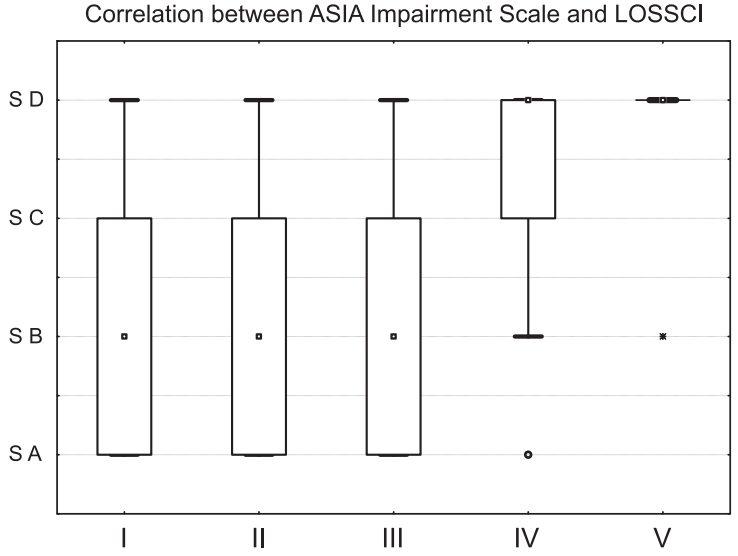

Figure 3 Box plot of the correlation between AIS and LOSSCI. Spearman's rank correlation coefficient $R$ is $0.30(P<0.05)$.

\section{Validity}

The validity data sets consist of 448 SCIM records from 161 patients $(2.8 \pm 0.9$ records per patient) obtained in the EMSCI time frames within the first year after injury (2 WAI: 20\%; 4 WAI: 31\%; 12 WAI: 31\%; 24 WAI: 16\%; 48 WAI: 1\%). Of these 161 patients, $65.8 \%$ were male, $54.7 \%$ were tetraplegic and $64.8 \%$ had an incomplete lesion. ISNCSCI data sets were available for 398 of the 448 SCIM records. Distributions of the AIS and NLI are shown in Figure 2. The correlation (Figure 3) between LOSSCI and AIS (coded as AIS $\mathrm{A}=1$, AIS $\mathrm{B}=2$, AIS $\mathrm{C}=3$ and AIS $\mathrm{D}=4)$ is weak $(R=0.30$, $P<0.05)$. Mean age at the time of injury was $48.3 \pm 20.2$ years, and the time between injury and testing was $66.9 \pm 56.5$ days. The mean difference of assessment dates (SCIM versus LOSSCI) was $5.7 \pm 4.4$ days (range $0-15$ ).

The distribution of LOSSCI stages is shown in Figure 4. LOSSCI stages are not uniformly distributed, because more than half of the records were determined as LOSSCI I. Spearman's correlation coefficients (all $P<0.0001)$ are listed in Table 2. Box plots (Figure 5) additionally depict the correlation between LOSSCI and all four SCIM subitems. The highest correlation was found between LOSSCI and SCIM indoor mobility (room and toilet; $R=0.82$ ). The lowest correlation was found between the SCIM respiration and sphincter management subscore and LOSSCI $(R=0.68)$.

\section{DISCUSSION}

The aim of this study was to apply and to adapt a rating scale, which was originally developed to categorize locomotor function in $\mathrm{CP}$, for the assessment of locomotor function in SCI and to quantify its reliability and validity. The adaption was necessary to reflect the different underlying pathologies of SCI. The original 10 LSs for CP were transformed into 5 stages to describe locomotor function in SCI. We found LOSSCI to have excellent reliability and moderate (SCIM subitem respiration and sphincter management) to strong (SCIM subitem self-care, mobility) validity. As expected, the SCIM mobility categories showed the best correlation. Indoor mobility displayed a slightly higher correlation (0.82) in comparison with outdoor mobility (0.77), indicating that LOSSCI better reflects indoor mobility aspects. The strong correlation with SCIM self-care category suggests that LOSSCI is not only suitable to categorize basic locomotor skills but also specific motor functions that are necessary for other everyday skills.

A complete LOSSCI assessment can be conducted by one trained physical therapist. It takes approximately $10 \mathrm{~min}$ and requires no
Locomotor Stages in Spinal Cord Injury (LOSSCI)

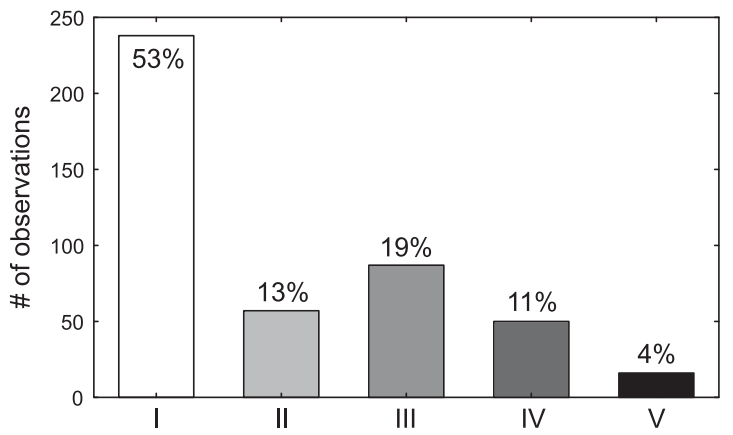

Figure 4 Distribution of LOSSCI stages of the validity cohort.

\section{Table 2 Spearman's $R$ of LOSSCI and SCIM items}

\begin{tabular}{lc} 
SCIM category & Spearman's R (all P $<0.0001$ ) \\
\hline SCIM 1: Self-care & 0.756 \\
SCIM 2: Respiration and sphincter management & 0.675 \\
SCIM 3a: Mobility indoors (room and toilet) & 0.816 \\
SCIM 3b: Mobility (indoor/outdoor) & 0.765 \\
SCIM 3a+3b & 0.798 \\
Total & 0.804
\end{tabular}

Abbreviations: LOSSCI, LOcomotor Stages in Spinal Cord Injury; SCIM, Spinal Cord Independence Measure.

technical aids besides a treatment table. LOSSCI is comparable to the WISCI assessment, ${ }^{23}$ which shows a high validity across multiple dimensions. ${ }^{8}$ However, testing the first five WISCI levels requires 10- m-long parallel bars, which are not available everywhere. The Spinal Cord Injury Functional Ambulation Profile (SCI-FAP) ${ }^{24}$ needs equipment that might not always be available, including a carpet, an armchair, two bricks, a trash can and four stairs with handrailing on both sides. Although designed for ambulatory individuals, the timed test can be applied in nonambulatory subjects by assigning the predefined maximum times for each of the seven individual timed items. Because of its nature, SCI-FAP is ideally suited as an outcome measure in the framework of interventional studies. However, it is not intended to be used for functional categorization of individuals with SCI. The Spinal Cord Injury Functional Ambulation Inventory $(\mathrm{SCI}-\mathrm{FAI})^{25}$ needs well-trained examiners and can only be assessed in already ambulating patients. This holds also true for the $10-\mathrm{m}$ walk test, the 6-min walk test and the timed up and go test. Besides these timed measures of ambulation, only SCIM, SCI-FAI, WISCI, ${ }^{26}$ SCI-FAP and the SCIM grades ${ }^{6}$ as categorical measures of ambulation are validated in the SCI population. The SCIM grades are constructed on a purely data-driven algorithm. In contrast, LOSSCI combines an initial data-driven approach with a final pathology- and expert-consensus-based approach to define and clinically describe five meaningful stages, which may help gain faster acceptance among clinicians. The AIS is an example of a coarse but clinically meaningful and well-accepted tool in spinal cord medicine. The found distributions of the ISNCSCI variables AIS and NLI in the validity trial reflect the analysis of a typical SCI cohort, ${ }^{27}$ which reinforces the generalizability of the obtained results. Although the distributions of LOSSCI and AIS look similar (Figures $2 \mathrm{~b}$ and 3 ), the weak correlation $(R=0.30)$ together with the strong correlation between SCIM and LOSSCI suggests that LOSSCI assesses-as intended-the functional 

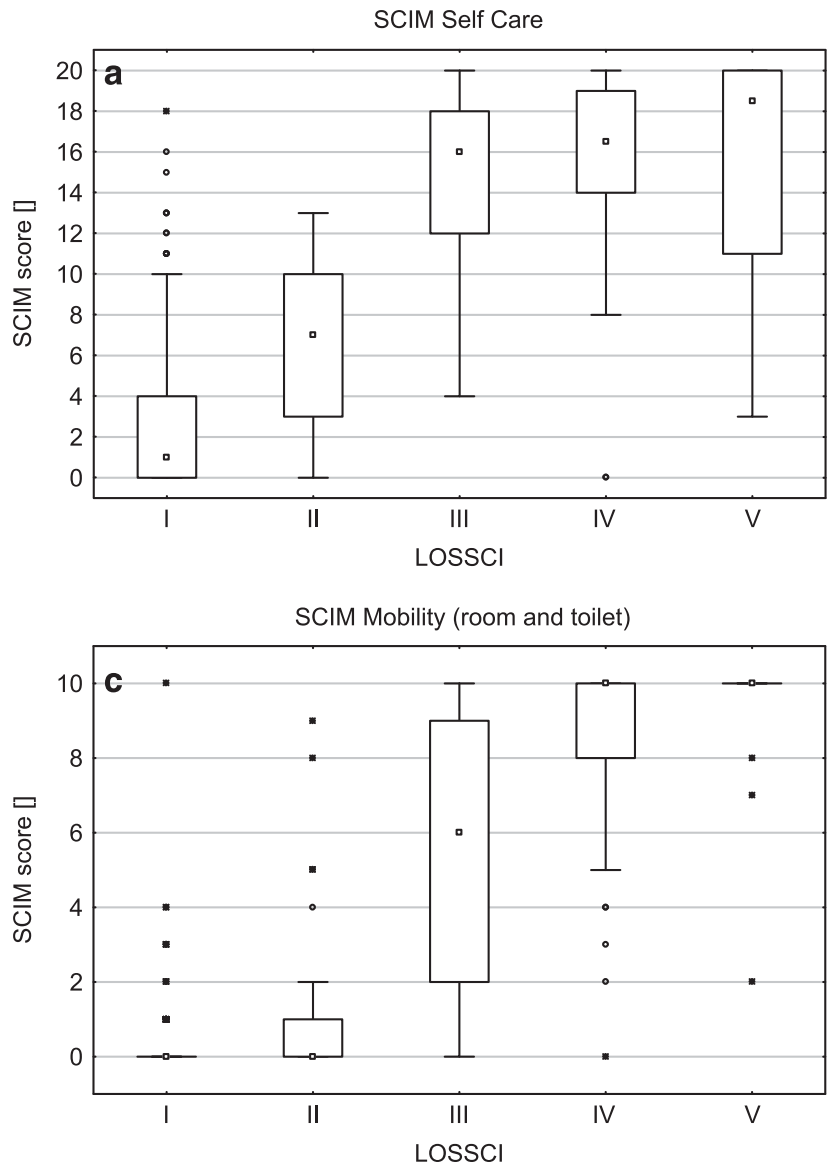

SCIM Respiration and sphincter management

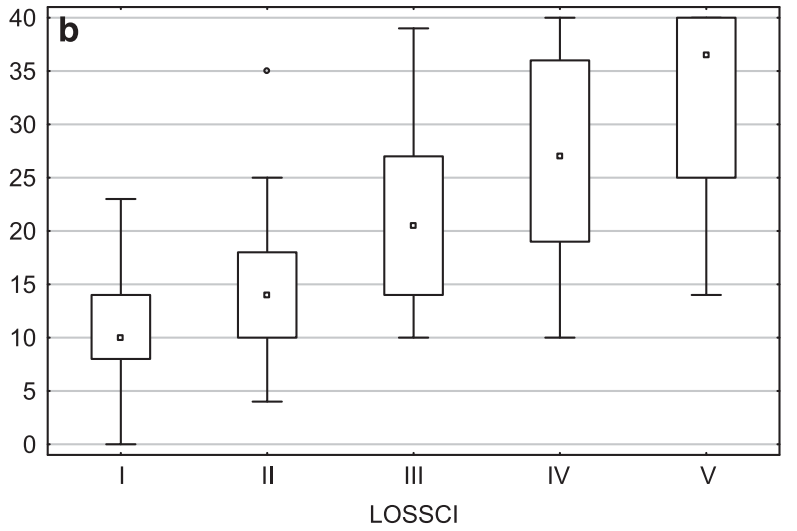

SCIM Mobility (indoor/outdoor)

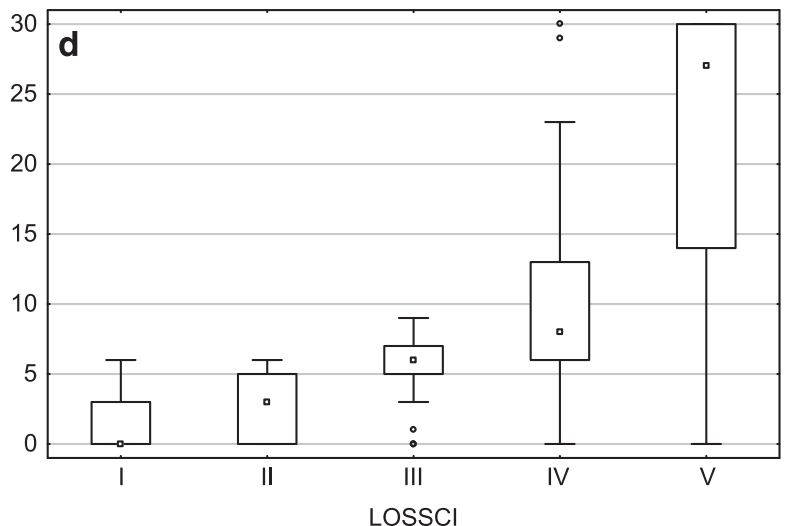

Figure 5 Box plots of the SCIM items: (a) self-care, (b) respiration and sphincter management, (c) mobility (room and toilet), and (d) mobility (indoor/outdoor) grouped by LOSSCI. Circles and crosses depict outliers and extremes, respectively.

capabilities of an individual with SCI. These are of course somehow related to the neurological impairment, but LOSSCI essentially assesses a different domain than the AIS.

\section{Study limitations}

The sample for the reliability part of this work represents predominately chronic SCI subjects, whereas acute SCI patients served as the basis for the validity study. The excellent reliability found in the chronic SCI patients might be generalized for an acute sample. However, the reliability will most likely be lower in acute SCI patients, because chronic, as opposed to acute, SCI patients have a rather stable locomotor pattern. ${ }^{23}$ The LOSSCI distribution in the validity cohort is skewed toward LOSSCI grade I (53\%, no trunk uprighting in prone position; Figure 3), which might reflect the contingent of tetraplegic subjects $(55 \%)$ in this study. However, the proportion of individuals with tetraplegia was not higher compared with other SCI cohorts. ${ }^{20}$ The low frequency (5\%) of ambulating patients might decrease the statistical power of the validity trial. This is most probably caused by an earlier discharge of ambulating subjects with incomplete SCI from the primary rehabilitation center, where all assessments were performed. Most of the assessments (51\%) were conducted within 4 WAI. During this period, the general functional skills of acute SCI patients are rather low compared with their neurological status. ${ }^{14} \mathrm{~A}$ second validation in a chronic SCI cohort would sort out this issue and would additionally address the different properties of acute and chronic SCI populations.
As LOSSCI is, by design, a coarse functional rating scale, some floor and ceiling effects are inevitable similar to in other locomotion assessments: for example, SCIM mobility subscale or WISCI. These effects are most noticeable in the box plots of Figure $5 c$, where the box of the lowest LOSSCI stage I collapsed (median $=25 \%$ quartile $=75 \%$ quartile) at the lowest possible SCIM scores and vice versa for the highest LOSSCI stage V. However, this collapsing effect is not present for the adjacent stages LOSSCI II and IV, indicating that there is no severe saturation effect. The goal of the iterative consensus process was to achieve a relatively uniform distribution between the LOSSCI stages, and Figure $5 \mathrm{c}$ indicates that this aim is fulfilled.

\section{Conclusion and future work}

LOSSCI provides a reliable and valid clinical tool to assess locomotor function in SCI. Locomotor function is reflected by a wide range of motor skills, not only bipedal walking as in most other assessments in SCI. The prognostic value of an early LOSSCI assessment with respect to neurological recovery (ISNCSCI), functional outcome (SCIM) and ambulation (walking tests) will be investigated in the future.

\section{DATA ARCHIVING}

There were no data to deposit.

\section{CONFLICT OF INTEREST}

The authors declare no conflict of interest. 


\section{ACKNOWLEDGEMENTS}

We thank all patients from Heidelberg and Bad Wildungen for participating in the project. We are grateful to Dr Tom Bruckner for his statistical support during the adaptation process.

1 Catz A, Itzkovich M, Steinberg F, Philo O, Ring H, Ronen J et al. The Catz-Itzkovich SCIM: a revised version of the Spinal Cord Independence Measure. Disabil Rehabil 2001; 23: 263-268.

2 Kalsi-Ryan S, Curt A, Verrier MC, Fehlings MG. Development of the Graded Redefined Assessment of Strength, Sensibility and Prehension (GRASSP): reviewing measurement specific to the upper limb in tetraplegia. J Neurosurg Spine 2012; 17: 65-76.

3 Frankel HL, Hancock DO, Hyslop G, Melzak J, Michaelis LS, Ungar GH et al. The value of postural reduction in the initial management of closed injuries of the spine with paraplegia and tetraplegia. I. Paraplegia 1969; 7: 179-192.

4 Kirshblum SC, Burns SP, Biering-Sorensen F, Donovan W, Graves DE, Jha A et al. International standards for neurological classification of spinal cord injury (revised 2011). J Spinal Cord Med 2011; 34: 535-546.

5 Catz A, Itzkovich M, Tesio L, Biering-Sorensen F, Weeks C, Laramee MT et al. A multicenter international study on the Spinal Cord Independence Measure, version III: Rasch psychometric validation. Spinal Cord 2007; 45: 275-291.

6 Bluvshtein V, Front L, Itzkovich M, Benjamini Y, Galili T, Gelernter I et al. A new grading for easy and concise description of functional status after spinal cord lesions. Spinal Cord 2012; 50: 42-50.

7 Dittuno PL, Ditunno JF Jr. Walking index for spinal cord injury (WISCI II): scale revision. Spinal Cord 2001; 39: 654-656.

8 Ditunno JF Jr., Ditunno PL, Scivoletto G, Patrick M, Dijkers M, Barbeau H et al. The Walking Index for Spinal Cord Injury (WISCI/WISCI II): nature, metric properties, use and misuse. Spinal Cord 2013; 51: 346-355.

9 Banaszek G. Vojta's method as the early neurodevelopmental diagnosis and therapy concept. Przegl Lek 2010; 67: 67-76.

10 Hungerford BA, Gilleard W, Moran M, Emmerson C. Evaluation of the ability of physical therapists to palpate intrapelvic motion with the Stork test on the support side. Phys Ther 2007; 87: 879-887.

11 Vojta V. Reflex rotation as a pathway to human locomotion. Z Orthop Ihre Grenzgeb 1970; 108: 446-452.
12 Shirley MM. The First Two Years: A Study of Twenty-five Babies, vol. J. University of Minnesota Press: Minneapolis, MN, USA, 1935

13 McGraw M. The neuromuscular maturation of the human infant. Yale J Biol Med 1943; 15: 925.

14 Curt A, Van Hedel HJ, Klaus D, Dietz V, EMSCI Study Group. Recovery from a spinal cord injury: significance of compensation, neural plasticity, and repair. J Neurotrauma 2008; 25: 677-685.

15 Lorenz DJ, Datta S, Harkema SJ. Longitudinal patterns of functional recovery in patients with incomplete spinal cord injury receiving activity-based rehabilitation. Arch Phys Med Rehabil 2012; 93: 1541-1552.

16 Fitzpatrick R, Davey C, Buxton MJ, Jones DR. Evaluating patient-based outcome measures for use in clinical trials. Health Technol Assess 1998; 2 i-iv 1-74.

17 Aaronson N, Alonso J, Burnam A, Lohr KN, Patrick DL, Perrin E et al. Assessing health status and quality-of-life instruments: attributes and review criteria. Qual Life Res 2002; 11: 193-205.

18 Ottenbacher KJ, Hsu Y, Granger CV, Fiedler RC. The reliability of the functional independence measure: a quantitative review. Arch Phys Med Rehabil 1996; 77. $1226-1232$.

19 Black TR. Evaluating Social Science Research: An Introduction. Sage Publications: London, UK, 1993.

$20 \mathrm{R}$ Core Team. R: A Language and Environment for Statistical Computing. R Foundation for Statistical Computing: Vienna, Austria, 2012 (http://www.R-project.org/).

21 Vojta V. On the prognosis of late treated children with cerebral palsy [Zur prognose der spät behandelnden CP kinder]. Der Kinderarzt 1987; 18: 1161-1172.

22 van Middendorp JJ, Pouw MH, Hayes KC, Williams R, Chhabra HS, Putz C et al. Diagnostic criteria of traumatic central cord syndrome. Part 2: a questionnaire survey among spine specialists. Spinal Cord 2010; 48: 657-663.

23 Burns AS, Delparte JJ, Patrick M, Marino RJ, Ditunno JF. The reproducibility and convergent validity of the walking index for spinal cord injury (WISCI) in chronic spinal cord injury. Neurorehabil Neural Repair 2011; 25: 149-157.

24 Musselman K, Brunton K, Lam T, Yang J. Spinal cord injury functional ambulation profile: a new measure of walking ability. Neurorehabil Neural Repair 2011; 25: 285-293.

25 Field-Fote EC, Fluet GG, Schafer SD, Schneider EM, Smith R, Downey PA et al. The Spinal Cord Injury Functional Ambulation Inventory (SCI-FAI). J Rehabil Med 2001; 33: $177-181$.

26 Lam T, Noonan VK, Eng JJ, Team SR. A systematic review of functional ambulation outcome measures in spinal cord injury. Spinal Cord 2008; 46: 246-254.

27 National Spinal Cord Injury Statistical Center, Spinal cord injury facts and figures at a glance. J Spinal Cord Med 2012; 35: 68-69. 